\title{
Assessing change in patient-reported quality of life after elective surgery: protocol for an observational comparison study [version 1; peer review: 2 approved]
}

\author{
Vanessa L. Kronzer', Michelle R. Jerry², Michael S. Avidan (iD1 \\ ${ }^{1}$ Department of Anesthesia, Washington University School of Medicine, Saint Louis, MO, 63110, USA \\ ${ }^{2}$ Department of Biostatistics, University of Michigan, Canton, MI, 48188, USA
}

\section{V1 First published: 24 May 2016, 5:976 \\ https://doi.org/10.12688/f1000research.8758.1}

Latest published: 24 May 2016, 5:976

https://doi.org/10.12688/f1000research.8758.1

\section{Abstract}

Despite their widespread use, the two main methods of assessing quality of life after surgery have never been directly compared. To support patient decision-making and study design, we aim to compare these two methods. The first of these methods is to assess quality of life before surgery and again after surgery using the same validated scale. The second is simply to ask patients whether or not they think their post-operative quality of life is better, worse, or the same. Our primary objective is to assess agreement between the two measures. Secondary objectives are to calculate the minimum clinically important difference (MCID) and to describe the variation across surgical specialties. To accomplish these aims, we will administer surveys to patients undergoing elective surgery, both before surgery and again 30 days after surgery. This protocol follows detailed guidelines for observational study protocols.

\section{Keywords}

quality of life, surgical procedures, outcome assessment , postoperative period

\section{Open Peer Review \\ Approval Status \\ 1 2 \\ version 1 \\ 24 May 2016
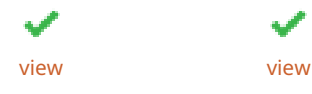 \\ 1. Anna Woodbury (D), Veteran Affairs Medical Center, Atlanta, USA \\ 2. Meghan Lane-Fall, University of \\ Pennsylvania Perelman School of Medicine, \\ Philadelphia, USA \\ Any reports and responses or comments on the article can be found at the end of the article.}


Corresponding author: Michael S. Avidan (avidanm@wustl.edu)

Competing interests: No competing interests were disclosed.

Grant information: VLK was supported by the Washington University Institute of Clinical and Translational Sciences grant UL1TR000448 from the National Center for Advancing Translational Sciences (NCATS) of the National Institutes of Health (NIH). MSA was supported by grant 1UH2AG050312-01 from the National Institute on Aging, grant BJHF\#7937-77 from the Barnes-Jewish Hospital Foundation, and the Washington University Department of Anesthesiology. The content is solely the responsibility of the authors and does not necessarily represent the official view of the NIH.

The funders had no role in study design, data collection and analysis, decision to publish, or preparation of the manuscript.

Copyright: $(2016$ Kronzer VL et al. This is an open access article distributed under the terms of the Creative Commons Attribution License, which permits unrestricted use, distribution, and reproduction in any medium, provided the original work is properly cited.

How to cite this article: Kronzer VL, Jerry MR and Avidan MS. Assessing change in patient-reported quality of life after elective surgery: protocol for an observational comparison study [version 1; peer review: 2 approved] F1000Research 2016, 5:976

https://doi.org/10.12688/f1000research.8758.1

First published: 24 May 2016, 5:976 https://doi.org/10.12688/f1000research.8758.1 


\section{Background}

The following protocol follows published guidelines for observational study protocols ${ }^{1}$. The research question for this study is how validated measures compare to self-reported measures of quality of life in patients undergoing elective surgery. To answer this question, we performed a literature search in PubMed.

Research studying patient-reported quality of life is burgeoning, including quality of life related to surgery ${ }^{2,3}$. Two main methods are used to estimate a procedure's impact on quality of life. The first is to compare patient scores before and after surgery using a validated quality of life scale ${ }^{4,5}$. The second method is to ask patients about their change in quality of life after surgery occurs ${ }^{6-8}$. This type of self-reported "global measure" is growing in popularity". However, patients' perceived change in quality of life may be inaccurate due to cognitive biases such as choice-supportive bias ${ }^{10,11}$, or theory-driven recall bias ${ }^{12,13}$. Uncovering potential bias in measures of quality of life is important since patients and clinicians base their surgical decision-making on these measures.

In addition, the minimum clinically important difference (MCID) in quality of life has been established in the literature for general populations $^{14,15}$ and for neurosurgical populations ${ }^{16,17}$, but has not been studied in general surgical populations. Establishing a MCID for quality of life is crucial, both for patients deciding whether or not to receive surgery and for clinicians evaluating the effectiveness of surgery.

With its large sample size and general population of elective surgery patients, this study is uniquely poised to compare the two methods of patient-reported change in quality of life and to determine the difference in quality of life score that surgical patients can detect. Determining the most accurate way to ascertain patient-reported quality of life can support elective surgery decisions and future studies of quality of life.

\section{Specific aims}

Aim 1

The primary aim of this study is to compare self-reported change in quality of life (better/same/worse) to the change in a validated (VR-12) quality of life score (both physical and mental component scores), 30 days after elective surgery.

We hypothesize that the median physical and mental quality of life scores will be significantly higher in patients reporting "better" quality of life compared to those reporting "same," and significantly higher in those reporting "same" quality of life to those reporting "worse." We also hypothesize that the overall agreement will be "substantial" (kappa=0.61 to 0.80), with the majority of error occurring in patients whose validated measure showed a decline in quality of life, but who reported "same" or "better" quality of life. For that reason, we expect the percent agreement between the self-reported and validated scales to be lowest in the group reporting "better" quality of life after surgery.
$\underline{\operatorname{Aim} 2}$

A secondary aim is to compare the change in physical and mental quality of life scores that patients were able to perceive to the MCID established in literature.

We hypothesize that the difference in quality of life that our surgical patients can detect will be similar to the difference reported in the literature (ie a 5-point change).

$\underline{\operatorname{Aim} 3}$

Another secondary aim is to describe the change in physical and mental quality of life for both methods across surgical specialties.

We hypothesize that the change in quality of life will be greater for specialties correcting limited problems such as orthopedic and plastic surgery, while the change will be lower for specialties with more complex problems such as neurosurgery and cardiothoracic surgery.

\section{Study design}

This prospective, observational cohort study is a sub-study of the Systematic Assessment and Targeted Improvement of Services Following Yearlong Surgical Outcomes Surveys (SATISFY-SOS) study. SATISFY-SOS is an ongoing registry that has been enrolling patients at Barnes Jewish Hospital since July, 2012 ${ }^{18}$. All enrolled patients complete a survey of baseline health during their visit to the preoperative assessment clinic and then complete a follow-up survey approximately 30 days after surgery (see Supplementary Material for these two surveys). The intervention for this study is to compare self-reported quality of life ("How would you rate your quality of life now? (better/same/worse)") to the quantitative change in their VR-12 quality of life scores between the baseline survey and 30-day follow-up survey. All patients answer both questions and therefore serve as their own controls.

\section{Study group}

The target population is all patients undergoing elective surgery at Barnes Jewish Hospital who attended the center for preoperative assessment and planning between January 15, 2014 and October 7, 2015. Inclusion criteria include age 18 or older, ability to read the English consent form (see Supplementary Material for the consent form), ability to consent, and plans to undergo elective surgery. Over $70 \%$ of patients undergoing elective surgery are assessed by the center for preoperative assessment and planning (CPAP) clinic before surgery. Reasons for no assessment include urgent surgery, geographical limitations, or surgeon preference. Approximately $65 \%$ of all eligible patients consent to participate in SATISFY-SOS. Reasons for non-participation include patient refusal ( $70 \%$ of cases), lack of nurse time or training $(20 \%)$, or lack of English literacy ( $10 \%)$. A study comparing participants to non-participants showed no major differences in characteristics ${ }^{19}$. Approximately $92 \%$ of consented patients complete the baseline survey, and $60 \%$ of those respond to the 30-day follow-up survey. 
A total of 9,097 "complete" records (with both baseline and 30-day surveys) are available in the proposed time window. For the purposes of this sub-study, only the first complete record for each will be included in the final dataset (approximately $94 \%$ of the available records). This practice ensures that each record is statistically independent from all the other records. In addition, records with surgery to 30-day response dates of less than 20 days or more than 120 days will be excluded.

\section{Recruitment}

Nurses at the CPAP clinic assess patient eligibility, recruit patients to participate, and obtain written consent. No payment is provided. While most patients decide whether or not to participate at this time, a patient can decide to participate any time between his or her CPAP visit and his or her surgery day. For patients who need special assistance, such as those who are blind or cannot physically sign a form, a witness can be obtained. However, in practice this rarely occurs. No arrangements are made for non-English speakers, mentally ill, children, or those suffering from dementia since those are excluded groups. If patients agree to participate, the CPAP nurse asks them to complete the baseline survey at the time of consent. Approximately 30 days following surgery, they receive a similar follow-up survey. Both surveys were designed to take 10 to 15 minutes to complete. The SATISFY-SOS research team holds monthly update meetings with all CPAP nurses to inform them about study progress and to encourage optimal recruitment.

\section{Data}

All preoperative and postoperative quality of life data comes from the SATISFY-SOS surveys, which are administered to patients at the preoperative assessment visit and then approximately 30 days after surgery. To maximize the follow-up survey response rate, patients are emailed the survey (once), mailed hard copy surveys (two times), and phoned (up to five times). The twelve items comprising the Veterans RAND 12 (VR-12) are items 24 through 35 on the survey, while the self-reported global quality of life question is item 1. The VR-12 is made up of two components: a physical component score (PCS), and a mental component score (MCS). Both scores are continuous on a scale from 0 to 100 (where higher is greater quality of life), and they are calibrated so that a score of 50 represents the US population mean ${ }^{20,21}$. The 30-day follow-up questionnaires also ask patients to self-report their change in quality of life. The question asks, "How would you rate your quality of life now?" with answer choices including "Better than before your procedure," "The same as before your procedure," and "Worse than before your procedure." Surgical specialty for the procedure is obtained from the electronic medical record. Using queries in MetaVision (iMDsoft, Needham, MA), the informaticist will provide the requested survey and medical record data to the investigators. He performs rigorous data validation on each queried variable.

SATISFY-SOS databases are hosted on a firewall-secured network server managed by the Department of Anesthesiology. The server is securely housed behind two locked doors within the departmental office suite and maintained and managed by the departmental IT team. Only the project Informaticist, Data Manager, and Director(s) have full access to these databases, which are also password-protected and encrypted for additional protection. Hardcopies of the baseline surveys are collected daily from the CPAP clinic and securely stored behind two locked doors within the Department of Anesthesiology. Baseline completed paper surveys are scanned into a digital image format (compressed TIFF). The digital image files are indexed and stored on a research file server that is attached to a private network with no public access. Survey email, mail and call lists are generated at Washington University in a similar manner to mailing lists for billing services. For each patient and date of service, a unique ID is generated and never duplicated. This unique ID is a nonsensical only meaningful to the research team.

Baseline surveys are processed by Solutions Data Systems. The digital image files are transmitted to Solutions Data Systems via secure file transfer protocol. When data entry has been confirmed, Solutions Data Systems deletes the digital image file from their servers. Press Ganey, a vendor specializing in patient survey distribution and collection, disseminates, collects, and processes 30-day and 1-year surveys. Paper surveys processed through automated scanning are all manually checked, and a manager listens to $10 \%$ of telephone surveys. All telephone surveys are recorded and available for future quality checks for performance improvement. Press Ganey stores the survey hardcopies for 90 days while the study team conducts spot-check quality assessments of the scanned data. The company then shreds the paper copies. Similarly, Press Ganey will hold copies of the electronic files and electronic recordings for 90 days, after which the electronic files are removed permanently from their system (and then only maintained by Washington University). During this 90-day period, the study team conducts additional quality assessments of the converted data.

\section{Statistical considerations}

We base sample size considerations for this study on the primary outcome. The first component of the primary endpoint is comparing the change in VR-12 quality of life scores among the three selfreported change groups (better/same/worse). Using a minimum important difference of five points, two tails, alpha of 0.05 , and $80 \%$ power, the required sample size is 77 patients per group, or 231 total patients among the three groups. The second component of the primary outcome is the agreement between the two quality of life measures, as reflected by the kappa statistic. Kappa does not have sample size requirements beyond lack of sparse cells. The third component of the primary outcome is comparing the percentage agreement across the three different self-reported change groups. Since no studies have performed this type of comparison previously, we pre-specified a $10 \%$ change as the minimum important change. Estimating $80 \%$ agreement, and using two tails, alpha $=0.05$, and $80 \%$ power, the required total sample size is 311 per group, or 933 total patients. Therefore, this study has adequate power for all of these endpoints.

The following statistical analyses will be performed, using alpha $=0.05$ and 95 percent confidence intervals, where appropriate. 
All analyses will be performed twice, once for VR-12 physical quality of life, and again for the VR-12 mental quality of life.

Aim 1:

- Compare change in VR-12 QOL scores for those answering better/same/worse (Kruskal-Wallis). If significant, will use Wilcoxon Rank-Sum tests to compare each of the three groups, using a Bonferroni correction of alpha $=0.017$.

- Calculate overall agreement between the self-reported and validated quality of life measures (using weighted kappa, which penalizes disagreements in proportion to their seriousness, see Table 1$)^{22}$.

- Calculate the overall percent of patients whose selfreported and validated quality of life scores matched (descriptive), including stratification by self-reported global change better/same/worse (compared using chi-square). For the purpose of this study, "matching" consists of:

o Change in VR-12 $>0$ for those responding their quality of life was "better"

o Change in VR-12 between +5 and -5 for those responding their quality of life was the "same"

o Change in VR-12 $<0$ for those responding their quality of life was "worse"

Aim 2:

- Calculate the quality of life where an equal proportion of patients reported better and same quality of life (MCID for improvement), or same and worse quality of life (MCID for deterioration). This is an anchor-based approach ${ }^{23}$.

Aim 3:

- Describe the change in VR-12 score and percent of patients reporting better, same, and worse quality of life for each of the following surgical specialties: neurosurgery, orthopedic, plastic, ophthalmologic, general, cardiac, gynecologic, otolaryngology, gastrointestinal/ hepatobiliary, urologic, and "other."

Only those answering both the self-reported quality of life question and at least ten out of twelve VR-12 questions at baseline and 30 days will be included. We will describe the characteristics of those with and without missing data. Multiple imputation will be used to fill in missing measurements for those missing two or fewer items on the VR-12 questionnaire. Analysis will be performed by VLK and MRJ after this protocol is submitted online.

\section{Limitations}

Although the "validated" VR-12 measure is based on a standardized scale that has been rigorously tested and studied, it is also self-reported and probably also contains bias. Because the VR-12 produces physical and mental component scores (PCS and MCS), but the self-reported question asks about overall quality of life, the observed association between the self-reported quality of life and the individual component scores might be artificially lowered. Also, the order of the questions on the surveys might influence patients' responses by priming them. Since the self-reported question occurs first, it may alter responses on the VR-12 items, which occur later in the same survey. Another limitation is that postoperative quality of life is only measured at 30 days. It is possible that the relationship between the two measures is different at different time points. In addition, the 30-day follow-up survey is often completed near 30 days, but the time of completion ranges from 20 to 120 days after surgery. Nevertheless, the time point for completing the two quality of life scales is the same in each individual patient, so the main results of this study should not be affected.

This study includes just one academic medical center, and its patient population and rules for preoperative assessment clinic attendance may differ from other hospitals. In addition, only $65 \%$ of eligible preoperative assessment clinic attendees enrolled in the study, which may introduce bias. However, our analyses indicate that participants do not differ in important ways from nonparticipants ${ }^{19}$. Furthermore, even if the sample was maximally biased, enrolling $65 \%$ of the actual target population means the results contain at least $80 \%$ accuracy ${ }^{24}$. Another factor that biases the sample is nonresponse to the follow-up surveys. Though we mitigate nonresponse through an extensive follow-up protocol, only $60 \%$ of patients respond to the 30-day survey. Fortunately, our previous work shows that the characteristics of responders do not differ significantly from the characteristics of non-responders (unpublished manuscript). Finally, anchor-based approaches for calculating MCID can be influenced by recall bias and have been shown to more strongly reflect patients' current health status than the true change over time ${ }^{23}$.

\section{Compliance}

Since the exposure for this study is patient-reported quality of life, no procedures for monitoring exposure compliance are necessary.

Table 1. Weights for weighted kappa calculation.

\begin{tabular}{|l|c|c|c|c|c|c|}
\hline & \multicolumn{5}{|c|}{$\begin{array}{c}\text { Change in VR-12 Score between } \\
\text { baseline and }\end{array}$} \\
\hline Self-days
\end{tabular}


Subjects are withdrawn from SATISFY-SOS if requested. The informaticist and Press Ganey are notified to ensure that the patient is no longer approached for data collection. As described in the consent form, data already collected may continue to be used.

\section{Ethical considerations}

This study is approved by the Institutional Review Board (Washington University Human Research Protection Office, IRB ID\# 201505035). As a sub-study of SATISFY-SOS, it has a waiver of informed consent. Written, informed consent is obtained from all participants for SATISFY-SOS (IRB ID\# 201203088). Since this study is survey-based, it involves no more than minimal risk to patients. As described above, no special allowances are made for non-English speakers, children, or mentally ill. Participants may withdraw from the study at any time.

\section{Finance and insurance}

Finance details, insurance details, and cover for negligent and non-negligent harm are not relevant for this study since it involves no more than minimal risk to patients. Patients receive no compensation for participation.

\section{Reporting and dissemination}

Results of this study will be presented at national meetings and published in a scientific journal. Participants will be individually notified of results only if discoveries are made that directly impact their health. The data and code for this project will be available upon email request.

Author contributions

VLK conceived the study protocol. MRJ provided statistical expertise. MSA provided SATISFY-SOS expertise. All authors were involved in the revision of the draft manuscript and have agreed to the final content.

\section{Competing interests}

No competing interests were disclosed.

Grant information

VLK was supported by the Washington University Institute of Clinical and Translational Sciences grant UL1TR000448 from the National Center for Advancing Translational Sciences (NCATS) of the National Institutes of Health (NIH). MSA was supported by grant 1UH2AG050312-01 from the National Institute on Aging, grant BJHF\#7937-77 from the Barnes-Jewish Hospital Foundation, and the Washington University Department of Anesthesiology. The content is solely the responsibility of the authors and does not necessarily represent the official view of the NIH.

The funders had no role in study design, data collection and analysis, decision to publish, or preparation of the manuscript.

\section{Supplementary Material}

\section{Baseline survey.}

Questionnaire provided to patients during their preoperative assessment visit

Click here to access the data.

\section{0-day survey.}

Follow-up questionnaire sent to patients approximately 30 days after surgery

Click here to access the data.

\section{Consent form.}

Consent form for SATISFY-SOS.

Click here to access the data. 
1. Guidelines for completing a research protocol for observational studies. Biostatistics Group, UCLH/UCL/RFH Biomedical Research Unit; 2010. Reference Source

2. Clancy C, Collins FS: Patient-Centered Outcomes Research Institute: the intersection of science and health care. Sci Transl Med. 2010; 2(37): $37 \mathrm{~cm} 18$. PubMed Abstract | Publisher Full Text

3. Waljee J, McGlinn EP, Sears ED, et al:: Patient expectations and patient-reported outcomes in surgery: a systematic review. Surgery. 2014; 155(5): 799-808. PubMed Abstract | Publisher Full Text | Free Full Text

4. Suk KS, Baek JH, Park JO, et al.: Postoperative quality of life in patients with progressive neuromuscular scoliosis and their parents. Spine J. 2015; 15(3): 446-453.

PubMed Abstract | Publisher Full Text

5. Tan TL, Le Duff MJ, Takamura KM, et al.: Do clinical and quality of life scores change over time after hip resurfacing? Hip Int. 2015; 25(2): 146-151. PubMed Abstract | Publisher Full Text

6. Skirko JR, Weaver EM, Perkins JA, et al.: Change in Quality of Life with Velopharyngeal Insufficiency Surgery. Otolaryngol Head Neck Surg. 2015; 153(5): 857-864.

PubMed Abstract | Publisher Full Text

7. Brown LK, Waljee AK, Higgins PD, et al:: Proximity to disease and perception of utility: physicians' vs patients' assessment of treatment options for ulcerative colitis. Dis Colon Rectum. 2011; 54(12): 1529-1536.

PubMed Abstract | Publisher Full Text

8. Boyd NF, Sutherland HJ, Heasman KZ, et al:: Whose utilities for decision analysis? Med Decis Making. 1990; 10(1): 58-67. PubMed Abstract | Publisher Full Text

9. Ubel PA, Loewenstein G, Jepson C: Disability and sunshine: can hedonic predictions be improved by drawing attention to focusing illusions or emotional adaptation? J Exp Psychol Appl. 2005; 11(2): 111-123. PubMed Abstract | Publisher Full Text

10. Smith D, Loewenstein G, Jepson C, et al:: Mispredicting and misremembering: patients with renal failure overestimate improvements in quality of life after a kidney transplant. Health Psychol. 2008; 27(5): 653-658. PubMed Abstract | Publisher Full Text

11. Mather M, Shafir E, Johnson MK: Misremembrance of options past: source monitoring and choice. Psychol Sci. 2000; 11(2): 132-138. PubMed Abstract | Publisher Full Text

12. Ubel PA, Loewenstein $\mathrm{G}$, Schwarz $\mathrm{N}$, et al: Misimagining the unimaginable: the disability paradox and health care decision making. Health Psychol. 2005; 24(4 Suppl): S57-62. PubMed Abstract | Publisher Full Text

13. Smith DM, Sherriff RL, Damschroder L, et al: Misremembering colostomies? Former patients give lower utility ratings than do current patients. Health Psychol.
2006; 25(6): 688-695.

PubMed Abstract | Publisher Full Text

14. Norman GR, Sloan JA, Wyrwich KW: Interpretation of changes in health-related quality of life: the remarkable universality of half a standard deviation. Med Care. 2003; 41(5): 582-592.

PubMed Abstract | Publisher Full Text

15. Farivar SS, Liu H, Hays RD: Half standard deviation estimate of the minimally important difference in HRQOL scores? Expert Rev Pharmacoecon Outcomes Res. 2004; 4(5): 515-523.

PubMed Abstract | Publisher Full Text

16. Zhou F, Zhang $Y$, Sun $Y$, et al.: Assessment of the minimum clinically important difference in neurological function and quality of life after surgery in cervical spondylotic myelopathy patients: a prospective cohort study. Eur Spine J. 2015; 24(12): 2918-2923.

PubMed Abstract | Publisher Full Text

17. Parker SL, Adogwa O, Mendenhall SK, et al.: Determination of minimum clinically important difference (MCID) in pain, disability, and quality of life after revision fusion for symptomatic pseudoarthrosis. Spine J. 2012; 12(12): 1122-1128. PubMed Abstract | Publisher Full Text

18. Avidan MS: Systematic Assessment and Targeted Improvement of Services Following Yearlong Surgical Outcomes Surveys (SATISFY-SOS).

In: ClinicalTrials.gov: [cited 2016 Feb 10]. Reference Source

19. Helsten DL, Abdallah AB, Avidan MS, et al.: Methodological considerations for collecting patient reported outcomes from unselected surgical patients. Anesthesiology. 2016; In Press.

20. Kazis LE, Miller DR, Skinner KM, et al.: Applications of methodologies of the Veterans Health Study in the VA healthcare system: conclusions and summary. J Ambul Care Manage. 2006; 29(2): 182-188. PubMed Abstract | Publisher Full Text

21. Selim AJ, Rogers W, Fleishman JA, et al.: Updated U.S. population standard for the Veterans RAND 12-item Health Survey (VR-12). Qual Life Res. 2009; 18(1): 43-52.

PubMed Abstract | Publisher Full Text

22. Sim J, Wright CC: The kappa statistic in reliability studies: use, interpretation, and sample size requirements. Phys Ther. 2005; 85(3): 257-268.

PubMed Abstract

23. Wright A, Hannon J, Hegedus EJ, et al:: Clinimetrics corner: a closer look at the minimal clinically important difference (MCID). J Man Manip Ther. 2012; 20(3): 160-166.

PubMed Abstract | Publisher Full Text | Free Full Text

24. Stamatopoulos C: Observations on the geometric properties of accuracy growth in sampling with finite populations. Rome: Food and Agriculture Organization of the United Nations; 1999.

Reference Source 


\section{Open Peer Review}

\section{Current Peer Review Status:}

\section{Version 1}

Reviewer Report 08 September 2016

https://doi.org/10.5256/f1000research.9425.r15800

(C) 2016 Lane-Fall M. This is an open access peer review report distributed under the terms of the Creative Commons Attribution License, which permits unrestricted use, distribution, and reproduction in any medium, provided the original work is properly cited.

\section{Meghan Lane-Fall}

Department of Anesthesiology and Critical Care, University of Pennsylvania Perelman School of Medicine, Philadelphia, PA, USA

In this report, Kronzer and colleagues detail a study protocol for an observational study of patient quality of life after elective surgery. They intend to use two methods to assess quality of life: (1) a validated scale and (2) a simple closed-ended question about whether their quality of life is better, worse, or the same as that before surgery. In the interest of research transparency, I applaud the authors on publishing their study protocol in advance of proceeding with their work. This will allow reviewers and readers of subsequent reports to determine whether the investigators adhered to their study protocol.

\section{Manuscript comments:}

The manuscript is clearly written and easy to understand. The data collection approach and analytic procedures are reasonable, and the authors have adequately addressed potential threats to validity (e.g. non-response bias). It is unfortunate that limited English speakers are excluded from the study population, but this is a limitation of the registry overall, not just this sub-study.

\section{Major criticisms:}

The authors state in their Limitations section that some of the surveys are completed as late as 120 days after surgery (instead of 30 days after). I worry that the relationship between the VR-12 score and the single global QOL question may not be consistent across time, which could bias the results. Have the authors considered restricting their sample to patients who complete the follow-up survey within a certain range of time (e.g. 20-40 days)? Minor criticisms:

I would define VR-12 earlier in the manuscript. It is currently in the Data section, but should be moved up to its first mention in the Methods section.

In the Data section, the authors state that the informaticist "performs rigorous data validation on each queried variable", but do not specify what the data validation procedures are. Some limited additional detail here would be useful. 


\section{Instrument comments:}

Minor criticism:

- In the baseline instrument, both "circle all" (Question 1) and "check all" (Question 2) are used - these should be the same for consistency.

\section{References:}

In Reference 19, the title is incorrect. "Methodological" should be "Methodologic", and the citation should be updated with publication information: Anesthesiology. 2016 Sep;125(3):495-504. doi: 10.1097/ALN.0000000000001217.

Competing Interests: No competing interests were disclosed.

\section{I confirm that I have read this submission and believe that I have an appropriate level of expertise to confirm that it is of an acceptable scientific standard.}

Reviewer Report 05 September 2016

https://doi.org/10.5256/f1000research.9425.r15801

(C) 2016 Woodbury A. This is an open access peer review report distributed under the terms of the Creative Commons Attribution License, which permits unrestricted use, distribution, and reproduction in any medium, provided the original work is properly cited.

\section{Anna Woodbury}

Department of Anesthesiology and Pain Management, Veteran Affairs Medical Center, Atlanta, GA, USA

The primary objective of this study is to perform a sub-study of the Systematic Assessment and Targeted Improvement of Services Following Yearlong Surgical Outcomes Surveys (SATISFY-SOS) study by comparing a validated quality of life measure (VR-12) to a self-reported "global" quality of life question. Secondary aims include comparing mental and physical quality of life pre- and postsurgery (Aim 2) and to describe these based on types of surgeries (Aim 3). The authors feel this is important because self-reported measures are subject to recall bias, and yet are more commonly used.

Major criticisms: None

Minor criticisms:

1. Co-morbidities (such as depression, Alzheimer's, or other psychiatric or neurologic illness) and their treatment or lack of treatment may affect quality of life scores. Will these be accounted for and will the patient be monitored? For example, if a patient suffers from depression, undergoes surgery, and is started on an antidepressant after surgery, this would potentially affect quality of life, but is not directly related to the surgery itself. I would like to know how this type of confounding variable will be addressed.

2. The validated tool used should be compared also with the PROMIS measures, which are 
essentially individually validated questions, often reliant on patient self-report. Would consider adding these.

Competing Interests: No competing interests were disclosed.

I confirm that I have read this submission and believe that I have an appropriate level of expertise to confirm that it is of an acceptable scientific standard.

\section{Comments on this article}

\section{Version 1}

\section{Reader Comment 19 Jul 2016}

David C. Norris, Precision Methodologies, LLC, USA

I would like to see Aim 3 pursued more vigorously, by disaggregating the category 'elective surgery' not across the coarse divisions of surgical specialty but according to more refined criteria that reflect deep theorizing based on detailed knowledge of the actual nature of the surgeries. Already as they posit Aim 3, the authors are revealing a construct like "get in there and fix/replace it" which appears applicable to certain surgeries (consider cataract surgery as a prime example) that address well defined problems and result in well defined improvement in function. Likewise, the authors seem to reveal an opposed construct like 'blunt' -- e.g., incompletely understood or unpredictable -- surgical interventions on complex types of functioning. (It does seem likely, as the authors intimate, that most neurosurgery falls into this category; but surely many surgeries in orthopedics will, too. Would bariatric surgery also serve as a prime example?) I conjecture that structured conversations with surgeons themselves will readily reveal examples of both categories of surgical intervention within each surgical specialty. I conjecture further that surgeons' affective response to surgeries on a Likert scale might correlate well with the degree of patient QoL improvement, especially with suitable 'priming' to intensify focus on the patient experience as opposed to the satisfactions derived from technical aspects of the surgeries.

One class of surgeries might deserve special attention in the authors' planned analyses: those for which the medical record would demonstrate objective measurement of functioning before and after. Again, cataract surgery (with its pre/post visual acuity measures) would be prototypical. This class of surgeries would enable the authors to investigate self-reported QoL changes in light of objective measures -- including in patient-level analyses.

Competing Interests: I report no competing interests. 
The benefits of publishing with F1000Research:

- Your article is published within days, with no editorial bias

- You can publish traditional articles, null/negative results, case reports, data notes and more

- The peer review process is transparent and collaborative

- Your article is indexed in PubMed after passing peer review

- Dedicated customer support at every stage

For pre-submission enquiries, contact research@f1000.com 